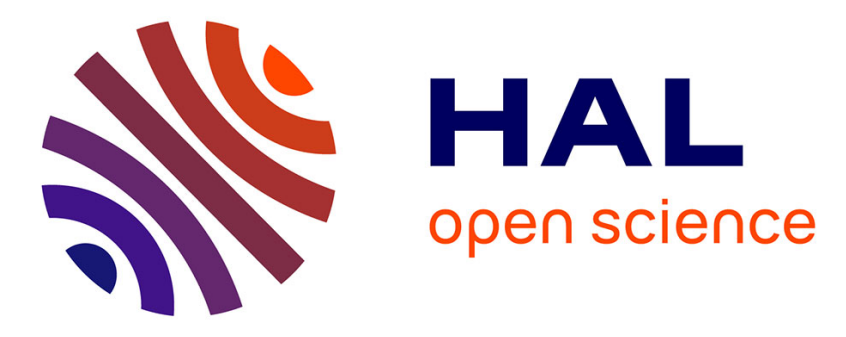

\title{
Homogenization of Moisture Diffusing Behavior of Composite Materials with Impermeable or Permeable Fibers
}

Djelloul Gueribiz, M. Rahmani, Frédéric Jacquemin, Sylvain Fréour, Ronald Guillen, K. Loucif

\section{To cite this version:}

Djelloul Gueribiz, M. Rahmani, Frédéric Jacquemin, Sylvain Fréour, Ronald Guillen, et al.. Homogenization of Moisture Diffusing Behavior of Composite Materials with Impermeable or Permeable Fibers: Application to Porous Composite Materials. Journal of Composite Materials, 2009, 43 (12), pp.1391-1408. 10.1177/0021998308104229 . hal-01006932

\section{HAL Id: hal-01006932 \\ https://hal.science/hal-01006932}

Submitted on 17 Jan 2017

HAL is a multi-disciplinary open access archive for the deposit and dissemination of scientific research documents, whether they are published or not. The documents may come from teaching and research institutions in France or abroad, or from public or private research centers.
L'archive ouverte pluridisciplinaire HAL, est destinée au dépôt et à la diffusion de documents scientifiques de niveau recherche, publiés ou non, émanant des établissements d'enseignement et de recherche français ou étrangers, des laboratoires publics ou privés.

\section{(c)(1)}

Distributed under a Creative Commons Attribution| 4.0 International License 


\title{
Homogenization of Moisture Diffusing Behavior of Composite Materials with Impermeable or Permeable Fibers - Application to Porous Composite Materials
}

\author{
D. GUERIBIZ AND M. RAHMANI \\ Département de Génie Mécanique, Université Amar Telidji Laghouat, Algérie \\ F. JACQUEMIN, S. FrÉOUR AND R. GUILLÉN \\ GeM - UMR CNRS 6183, Université de Nantes, France \\ K. LOUCIF \\ Département de mécanique de précision et optique \\ Université Ferhat Abbas Sétif, Algérie
}

\begin{abstract}
In order to predict the long-term durability of polymer matrix composite materials submitted to humid environments, the moisture diffusion behavior has to be investigated. The knowledge of the effective diffusivity is actually required, for estimating the moisture content of polymer-based fiber-reinforced materials, even when a basic behavior such as Fick's law is assumed to occur. The original contribution of the present work is to provide new analytical solutions for the effective diffusivities from the solving of unit cell problems on representative volume elements by means of several multi-scale approaches. Composite materials with impermeable or permeable fibers are extensively investigated. The proposed approaches are extended to the practical case of composite materials containing realistic voids volume fractions.
\end{abstract}

KEY WORDS: homogenization, effective moisture diffusivity, closed-form solutions.

\section{INTRODUCTION}

COMPOSITE MATERIALS ABSORB moisture during their service life, principally through the resin, and also through the fibers when they are permeable [1]. In numerous cases, the moisture kinetic follows the classical Fick's law, according to Springer and Shen [2] 
and Jacquemin and Vautrin [3]. The knowledge of the moisture diffusivity and the appropriate boundary conditions enables calculating the time-dependent moisture content field in the composite material, which can be useful for determining the corresponding mechanical states, for instance. The purpose of this article is to propose original analytical solutions for the effective moisture diffusivity of polymeric composite materials with impermeable or permeable fibers, through several appropriate scale-transition models. The effective diffusivity is deduced from solving a unit cell problem on a representative volume element (RVE). The defined RVE is submitted to a macroscopic moisture gradient inducing local fields and gradients within the RVE. The effective diffusivity is calculated by considering the discontinuity of the moisture contents and the continuity of the moisture flux that occur at the boundary between the composite plies constituents. At the interface between two materials exhibiting heterogeneous moisture diffusion behavior, only the continuity of the chemical potentials is actually assumed to be satisfied [4]. Thus, a parameter representative of the gap in terms of moisture content experienced by two adjacent constituents is introduced in the unit cell problem to be solved and therefore appears in the proposed expressions for the effective diffusivities. In the case of permeable fibers, inverse approaches enable the determination of the moisture diffusivity of the fibers. A comparison with effective diffusivities calculated according to finite element methods is also achieved. Finally, the presented multi-scale approaches are extended to porous composite materials so that the influence of void contents on the effective diffusivity would also be discussed.

\section{EFFECTIVE MOISTURE DIFFUSIVITY}

The multi-scale approaches, considered in the present work, are based on the solving of unit cell problems on various RVE. Several approaches are used in order to estimate the effective moisture diffusivity of fiber-reinforced polymer matrix composite plies: a threephase model, a self-consistent model, and a finite element approach.

\section{Three-phase Model}

The RVE (Figure 1) is composed of the reinforcing fiber, the polymer matrix, and the equivalent homogeneous medium, the properties of which have to be determined. The fiber radius and volume will be denoted by $r_{f}$ and $V_{f}$, respectively, whereas the

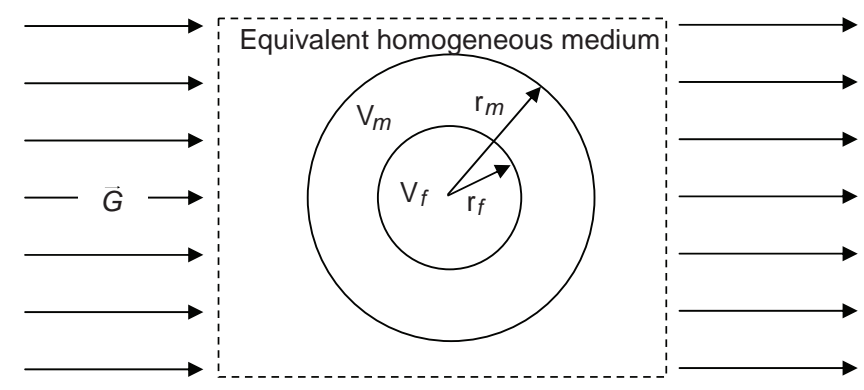

Figure 1. Representative volume element. 
corresponding quantities, for the matrix, will be denoted by $r_{m}$ and $V_{m}$. In this configuration, the application of a moisture content flux $\vec{G}$ on the RVE enables to obtain the equivalent diffusing behavior.

The unit cell problem is defined by the following system of equations:

$$
\left\{\begin{array}{l}
\operatorname{div} \vec{q}=0 \\
\vec{q}=-D \overrightarrow{\operatorname{grad}} C \\
\|\vec{q} \cdot \vec{n}\|=0 \text { at the interface fiber/matrix } \\
\|C\| \neq 0 \text { at the int erface fiber/matrix }
\end{array}\right.
$$

where $\vec{q}$ is the moisture content flux, $\vec{n}$ the normal vector, and $D$ the diffusion coefficient.

The sign \|\| stands for the moisture contents and flux at the interfaces. For cylindrical constituents,

$$
\operatorname{div} \vec{q}=\operatorname{div}(-D \overrightarrow{\operatorname{grad}} C)=-D\left(\frac{1}{r} \frac{\partial C}{\partial r}+\frac{\partial^{2} C}{\partial r^{2}}+\frac{1}{r^{2}} \frac{\partial^{2} C}{\partial \theta^{2}}\right)
$$

and the moisture content field solution of the unit cell problem (1) reads ( $r$ and $\theta$ standing for the radial and circumferential coordinates, respectively):

$$
\left\{\begin{array}{ll}
C(r, \theta)=A_{f} r \cos (\theta) & \text { in the fiber } \\
C(r, \theta)=\left(A_{m} r+\frac{B_{m}}{r}\right) \cos (\theta) & \text { in the matrix } \\
C(r, \theta)=G r \cos (\theta) & \text { in the equivalent medium }
\end{array},\right.
$$

where $A_{f}, A_{m}, B_{m}$, and $G$ are unknown constants.

Let us consider, (i) the boundary conditions, (ii) the continuity and discontinuity conditions at the interfaces, and (iii) the permeability of the fibers.

At $r=r_{m}$

The continuity of the moisture contents at the boundary between the equivalent medium and the matrix is considered:

$$
A_{m} r_{m}^{2}+B_{m}-G r_{m}^{2}=0
$$

The moisture content flux in the matrix is equal to the moisture content flux in the equivalent medium, so that

$$
\left.D_{m} \frac{\partial C}{\partial r}\right|_{r_{m}}=\left.D_{e f f} \frac{\partial C}{\partial r}\right|_{r_{m}}
$$

thus:

$$
D_{m} A_{m} r_{m}^{2}-D_{m} B_{m}-D_{e f f} G r_{m}^{2}=0
$$

where $D_{m}$ and $D_{\text {eff }}$ are the diffusion coefficients of the matrix and the equivalent medium, respectively. 
At $r=r_{f}$

The continuity of the flux between fiber and matrix,(which writes $\left.D_{f} \frac{\partial C}{\partial r}\right|_{r_{f}}=\left.D_{m} \frac{\partial C}{\partial r}\right|_{r_{f}}$ ), is considered:

$$
D_{m} A_{m} r_{f}^{2}-D_{m} B_{m}-D_{f} A_{f} r_{f}^{2}=0
$$

where $D_{f}$ is the diffusion coefficient of the fiber.

The discontinuity of the moisture contents between fiber and matrix involves a moisture content gap: $C_{f}=\alpha C_{m}$, so that the following relation has to be satisfied:

$$
\alpha A_{m} r_{f}^{2}+\alpha B_{m}-A_{f} r_{f}^{2}=0
$$

where $\alpha$ is a constant representative to the moisture content gap between fiber and matrix ( $\alpha$ can be defined if and only if $C_{m} \neq 0$ i.e., for a composite having a permeable matrix).

The Equations (3)-(6) constitute a system of four equations with four unknown constants $\left(A_{m}, B_{m}, A_{f}, G\right)$ :

$$
\left[\begin{array}{cccc}
r_{m}^{2} & 1 & -r_{m}^{2} & 0 \\
D_{m} r_{f}^{2} & -D_{m} & 0 & -D_{f} r_{f}^{2} \\
D_{m} r_{m}^{2} & -D_{m} & -D_{e f f} r_{m}^{2} & 0 \\
\alpha r_{f}^{2} & \alpha & 0 & -r_{f}^{2}
\end{array}\right]\left[\begin{array}{c}
A_{m} \\
B_{m} \\
G \\
A_{f}
\end{array}\right]=\left[\begin{array}{l}
0 \\
0 \\
0 \\
0
\end{array}\right] .
$$

In order to obtain a set of nontrivial solutions for the unknown constants, the mathematical condition is that the determinant of the matrix presented in (7) must be equal to zero. Thus, making this determinant equal to zero, the effective diffusion coefficient is obtained:

$$
D_{e f f}=D_{m} \frac{D_{m}\left(1-\left(r_{f}^{2}\right) /\left(r_{m}^{2}\right)\right)+\alpha D_{f}\left(1+\left(r_{f}^{2}\right) /\left(r_{m}^{2}\right)\right)}{D_{m}\left(1+\left(r_{f}^{2}\right) /\left(r_{m}^{2}\right)\right)+\alpha D_{f}\left(1-\left(r_{f}^{2}\right) /\left(r_{m}^{2}\right)\right)} .
$$

For a cylindrical geometry of the RVE, the ratio $r_{f}^{2} / r_{m}^{2}$ corresponds to the fiber volume fraction $v_{f}$. As a consequence, the expression (8) can be expressed as follows:

$$
\frac{D_{e f f}}{D_{m}}=\frac{\left(1-v_{f}\right)+\alpha\left(D_{f} / D_{m}\right)\left(1+v_{f}\right)}{\left(1+v_{f}\right)+\alpha\left(D_{f} / D_{m}\right)\left(1-v_{f}\right)}
$$

Starting from the knowledge of $D_{\text {eff }}$ and $D_{m}$, Equation (9) enables the determination of $D_{f}$. By introducing the parameter $\psi=\alpha \frac{\left(D_{f}\right)}{\left(D_{m}\right)}$, expression (9) becomes:

$$
\frac{D_{e f f}}{D_{m}}=\frac{\left(1-v_{f}\right)+\psi\left(1+v_{f}\right)}{\left(1+v_{f}\right)+\psi\left(1-v_{f}\right)} .
$$


In the particular case, when $\psi=0$, we get the classical Hashin's expression [5] for composite materials with impermeable fibers:

$$
\frac{D_{e f f}^{i m p r}}{D_{m}}=\frac{1-v_{f}}{1+v_{f}} .
$$

Figure 2 shows the evolution of the effective diffusion coefficient, obtained through Equation (10), as a function of the parameter $\psi$, for different fiber volume fractions. In this representation, the parameter $\psi$ can be assimilated to a fiber permeability index. A fiber permeability index lower than one involves that the fibers reduce the velocity of the diffusion process in the composite material (the most frequently encountered case in practice), by comparison to the reference diffusive behavior of the neat resin.

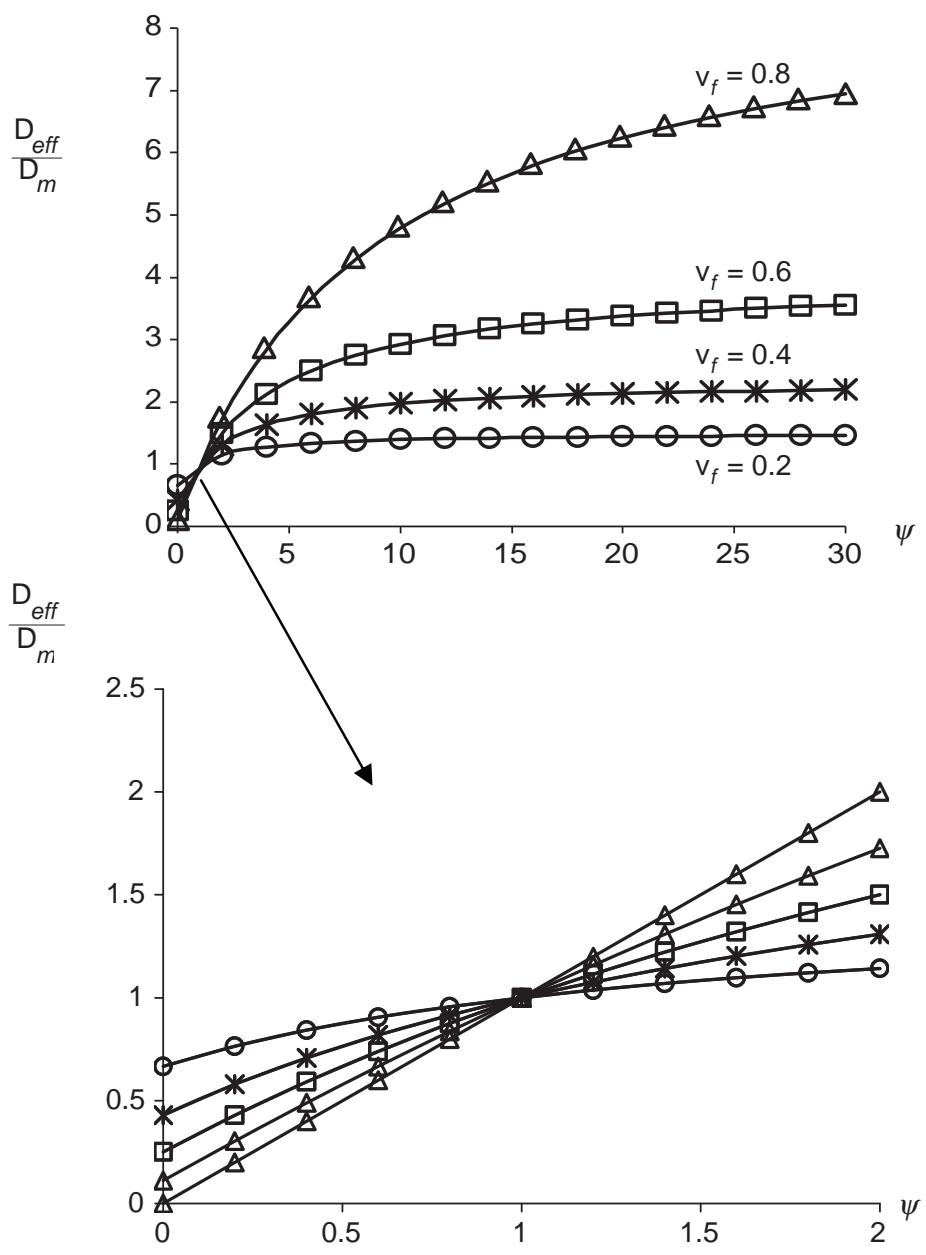

Figure 2. Dimensionless effective diffusivity as a function of $\psi$. 
For a material constituted by a quasi-impermeable matrix, on the one hand, and by fibers presenting a strong permeability, on the other hand, $\psi$ should be higher than 1 . As a consequence, this representation, featuring the parameter $\psi$, enables to consider numerous diffusive behaviors.

The diffusion coefficient is a growing function of the parameter $\psi$. It nevertheless tends towards a constant level for a value of $\psi$ depending on the fiber volume fraction $v_{f}$. The intersection of the displayed curves, observed at $\psi=1$, actually corresponds to $D_{\text {eff }}=D_{m}$. The regions with $\psi<1$ and $D_{\text {eff }}<D_{m}$ characterize composite materials whose fibers present a weak permeability. The fact that the curves with stronger reinforcements volume fractions are below those with weaker fibers volume fractions reveals the attenuation effect due to the fibers on the moisture diffusion process.

\section{Self-consistent Model}

In this model, the fiber, that fills a volume $V_{f}$, is embedded in an infinite matrix, which takes up a volume $V_{\text {eq }}$, and has the researched equivalent diffusive properties (Figure 3).

In this configuration, the application of a moisture content flux $\vec{G}$ on the RVE enables to obtain the equivalent diffusing behavior.

The moisture content field solution of the unit cell problem (satisfying $\operatorname{div} \vec{q}=0$ ) is:

$$
\left\{\begin{array}{ll}
C(r, \theta)=A_{f} r \cos (\theta) & \text { in } V_{f} \\
C(r, \theta)=\left(G r+\frac{B_{a c}}{r}\right) \cos (\theta) & \text { in } V_{e q}
\end{array},\right.
$$

where $A_{f}, B_{a c}$, and $G$ are unknown constants.

The homogeneous equivalent behavior is defined as follows:

$$
D_{\text {eff }}<\overrightarrow{\operatorname{grad}} C(y)>=v_{f} D_{f}<\overrightarrow{\operatorname{grad}} C(y)>_{V_{f}}+v_{m} D_{m}<\overrightarrow{\operatorname{grad}} C(y)>V_{m},
$$

where the quantity $\langle\overrightarrow{\operatorname{grad}} C(y)\rangle_{V}$ denotes the average of the quantity $\overrightarrow{\operatorname{grad}} C(y)$ over the volume $V$ as follows:

$$
\langle\overrightarrow{\operatorname{grad}} C(y)\rangle_{V}=\frac{1}{|V|} \int_{\partial V} C \vec{n} d s,
$$

$\partial V$ is the outer surface delimiting $V$, whereas $\vec{n}$ is the unit vector normal to this border.

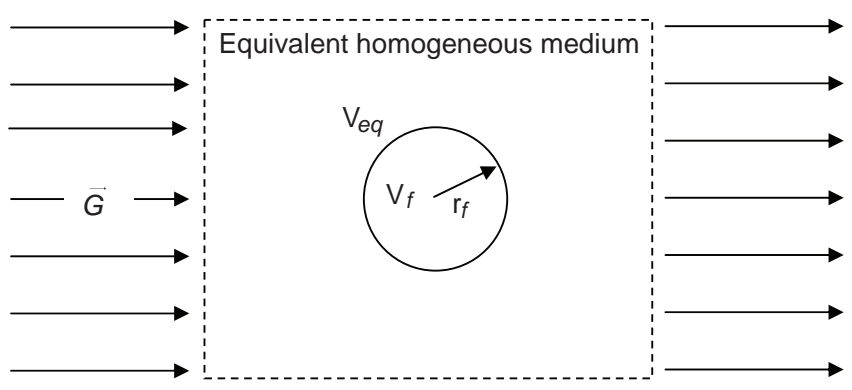

Figure 3. Self-consistent model (RVE). 
Finally, Equation (13) and the interface conditions lead to the following system of equations:

$$
\left[\begin{array}{ccc}
r_{f}^{2} & 1 & -r_{f}^{2} \\
D_{e f f} r_{f}^{2} & -D_{e f f} & -D_{f} r_{f}^{2} \\
D_{e f f}-D_{m} & 0 & v_{f}\left(D_{m}-D_{f}\right)
\end{array}\right]\left[\begin{array}{c}
G \\
B_{a c} \\
A_{f}
\end{array}\right]=\left[\begin{array}{l}
0 \\
0 \\
0
\end{array}\right]
$$

Equating the determinant of expression (14) to zero, one obtains the following equation of the second degree in $D_{e f f}$ :

$$
D_{e f f}^{2}+D_{e f f}\left[D_{m}\left(2 v_{f}-1\right)+D_{f}\left(1-2 v_{f}\right)\right]-D_{f} D_{m}=0 .
$$

It is easy to check that, the single positive root of (15) satisfies:

$$
\frac{D_{e f f}}{D_{m}}=\frac{1}{2}\left(\frac{D_{f}}{D_{m}}\left(2 v_{f}-1\right)+\left(1-2 v_{f}\right)+\sqrt{\frac{4 D_{f}}{D_{m}}+\left[\frac{D_{f}}{D_{m}}\left(1-2 v_{f}\right)+\left(2 v_{f}-1\right)\right]^{2}}\right) .
$$

For an impermeable fiber (i.e., introducing the condition $D_{f}=0$ ) the normalized effective diffusivity simplifies as follows:

$$
\frac{D_{\text {eff }}}{D_{\mathrm{m}}}=1-2 v_{f}
$$

The domain of validity of expression (17) is limited to $v_{f}$ values lower than 0.5 . In order to improve the results of this model and to extend its domain of validity to stronger fiber volume fractions, the self-consistent model was associated to an iterative calculation procedure (IP), following the approach detailed in references [6,7]. The final fiber volume fraction is reached by introducing step by step a partial volume fraction $f_{p}\left(f_{p}=v_{f} / n\right.$ where $n$ is the number of iterations).

Figure 4 reports the results obtained with the self-consistent model associated, or not, to the iterative calculation process, which are compared to the three-phase approach.

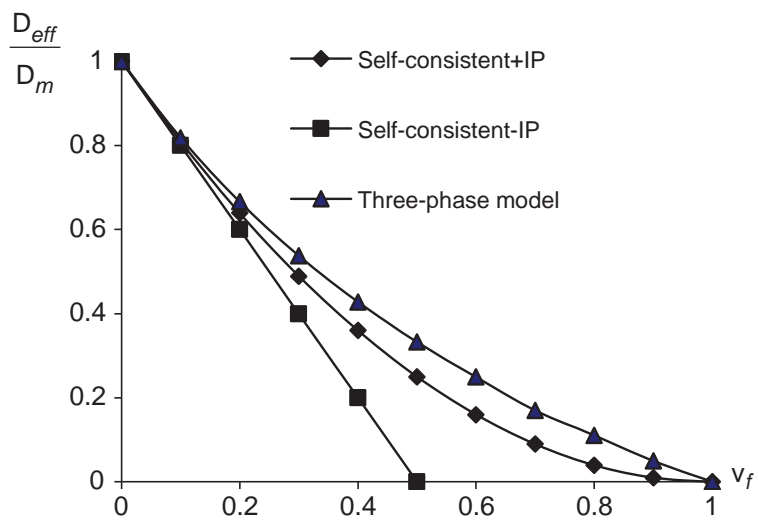

Figure 4. Effective diffusion coefficient deduced from the self-consistent model coupled and not coupled to iterative process (IP). 
The results of the self-consistent model become closer to those provided by the three-phase model, particularly for weak fiber volume fraction, when the iterative calculation procedure is followed.

\section{Comparison with Numerical Approaches}

Numerical methods based on a resolution of the cellular problem by finite elements [8] or by finite differences can be used in order to characterize the diffusive behavior of composites materials. The considered unit cells are bidimensional and symmetrical. The resolution of the cellular problem provides the moisture content field in the unit cell and the effective diffusion coefficient is determined from the relation:

$$
D_{e f f}\langle\overrightarrow{\operatorname{grad}} C(y)\rangle=D_{e f f} \vec{G}=v_{f} D_{f}\langle\overrightarrow{\operatorname{grad}} C(y)\rangle_{V_{f}}+\left(1-v_{f}\right) D_{m}\langle\overrightarrow{\operatorname{grad}} C(y)\rangle_{V_{m}},
$$

where $\langle\overrightarrow{\operatorname{grad}} C(y)\rangle_{V_{m}}$ and $\langle\overrightarrow{\operatorname{grad}} C(y)\rangle_{V_{f}}$ are the average concentration gradients over the volume of the matrix and that of the fiber, respectively.

\section{CASE OF IMPERMEABLE FIBERS}

Let us first consider a unidirectional composite with impermeable fibers. Such a composite exhibits a transversely isotropic behavior with regard to moisture diffusion. In order to evaluate the effective diffusivity of the unit cell, it is sufficient to solve a stationary 1D diffusion problem featuring Dirichlet-type boundary conditions on the two opposite edges (corresponding to a uni-axial concentration gradient $G$ ). The flux in the other directions is considered to be null. Since the fiber is impermeable, the flux will fall to zero in its vicinity [9]:

$$
\frac{\partial C}{\partial n}=0
$$

where $\vec{n}$ is the normal vector to the surface of the fiber (Figure 5).

The cellular problem, corresponding to square or hexagonal arrangements of the fibers (Figure 5), was achieved through the finite element method by using Matlab software. Figure 6 shows the resulting reduced effective diffusivity as a function of the fiber volume fraction for hexagonal and square arrangements and those obtained according to the three-phase model (11). No difference occurs for the reduced diffusivity, whatever the considered fiber arrangement, for reinforcements volume fractions included in the range $0-40 \%$. Moreover, in this range of fiber volume fractions, the numerical approaches are close to the results obtained through the three-phase model. For reinforcement volume fractions above $40 \%$, the results provided by the numerical approach significantly depends on the considered geometrical arrangement for the unit cell. The discrepancy between the estimated effective diffusivities increases as a function of the fiber volume fraction. Only the numerical predictions obtained by assuming a hexagonal array of fibers remain close to those provided by the three-phase model (up to a fiber volume fraction of 0.8 ). The numerical value for diffusivity is zero for a fiber volume fraction equal to $0.785(\pi / 4)$, which represents the upper limit value of reinforcement for a square array, whereas for a hexagonal arrangement, this value is close to $0.9(\pi /(2 \sqrt{3}))$. Only, the analytical approach enables to calculate the reduced effective diffusivity for $v_{f}=1$ (this should nevertheless be considered as a purely theoretical situation, without practical applications). 


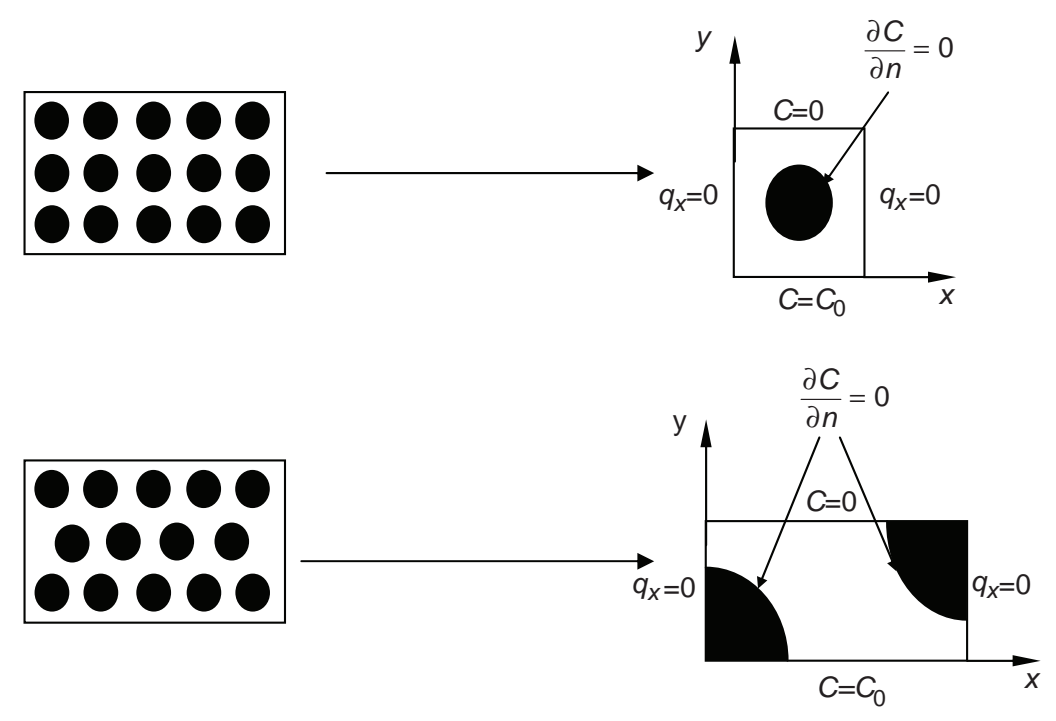

Figure 5. Square and hexagonal arrangement of the fibers.

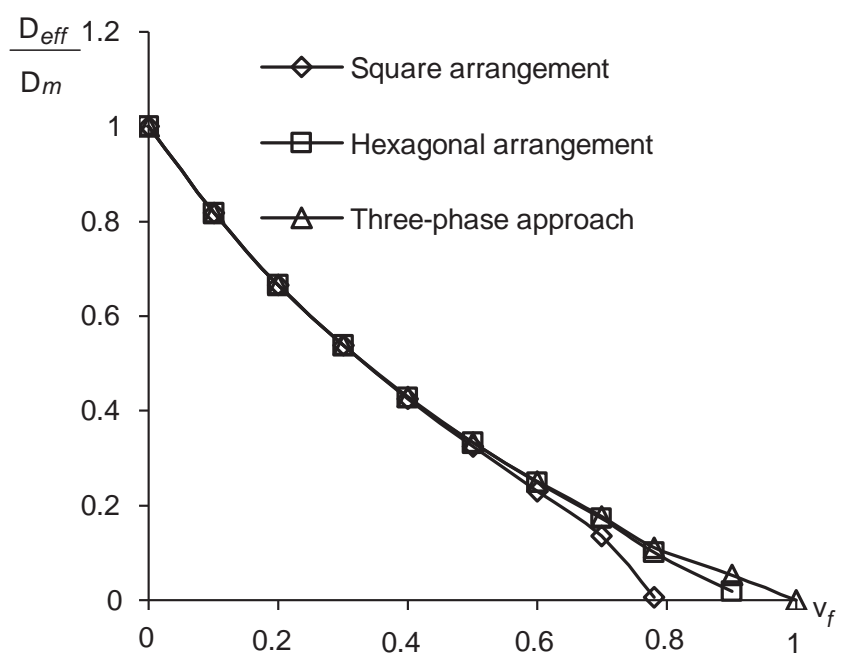

Figure 6. Numerical approaches for composites with impermeable fibers.

\section{CASE OF PERMEABLE FIBERS}

In the case of a unidirectional composite with permeable fibers, diffusion happens through two media: the matrix and the fibers, the diffusivities of which are strongly heterogeneous. As stated earlier at the time of the analytical solving of the cellular problem, there still exists a concentration gap at the fiber/matrix interface.

In order to find a numerical solution to this problem, spatial and temporal finite difference discretization is required. The numerical scheme used here is explicit.

Figure 7 shows the reduced effective diffusivity obtained by the actual numerical approach and the analytical expression (10). The obtained values are very similar, particularly for weak fiber volume fractions. 


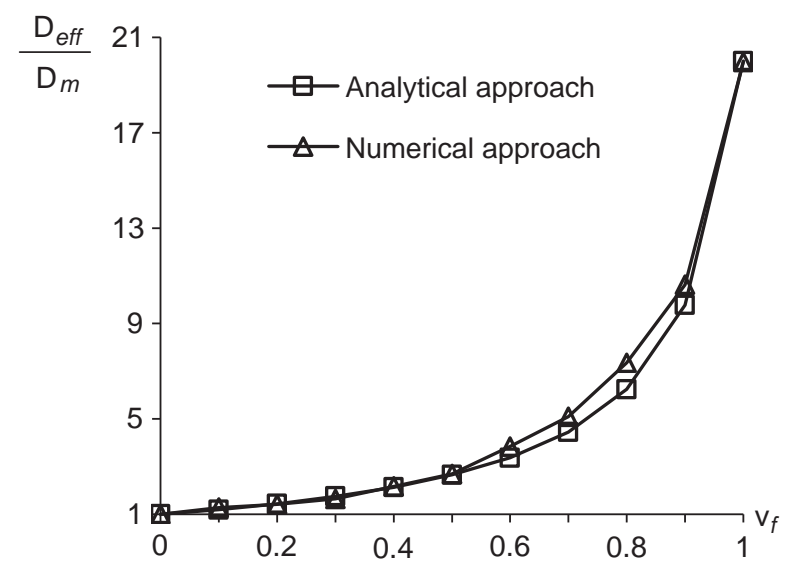

Figure 7. Reduced effective diffusivity obtained from analytical and numerical approaches $(\psi=20)$.

\section{EFFECTIVE MOISTURE DIFFUSIVITY OF POROUS COMPOSITE MATERIALS}

The presence of voids in composite materials, is known to induce significant decrease of the mechanical properties, even at very weak volume fraction [10,11]. Moreover, in humid environment they also affect the moisture absorption [12,13]. The aim of this part is to investigate the effect of voids on the moisture diffusion process in composite materials.

\section{Composite Materials with Porous Matrix}

We assume a perfect adhesion between fibers and matrix and that the voids are only embedded in the matrix. Therefore, the first step is to determine the effective diffusion coefficient of the porous matrix.

A moisture content gap, denoted by parameter $\alpha_{m v}$, is introduced at the interface between matrix and voids. The volume fractions of voids are often weak (i.e., lower than $5 \%$ ) in composite materials, so that the diluted distribution approach, illustrated by the RVE presented in Figure 8, can be used. In this approach, the application of a moisture content flux $\vec{G}$ on the RVE enables to obtain the equivalent diffusing behavior.

The moisture content field solution of the unit cell problem is:

$$
\begin{cases}C(r \theta)=A_{v} r \cos (\theta) & \text { in the fiber } \\ C(r \theta)=\left(G r+\frac{B_{m}}{r}\right) \cos (\theta) & \text { in the matrix }\end{cases}
$$

where $A_{v}, B_{m}$, and $G$ are unknown constants.

The homogeneous equivalent behavior is defined as follows:

$$
D_{e f f}^{m}\langle\overrightarrow{\operatorname{grad}} C(y)\rangle=v_{v} D_{v}\langle\overrightarrow{\operatorname{grad}} C(y)\rangle_{V_{v}}+v_{m} D_{e f f}^{m}\langle\overrightarrow{\operatorname{grad}} C(y)\rangle_{V_{m}},
$$

where $v_{v}$ is the volume fraction of voids whereas $D_{v}$ denotes their diffusivity. 


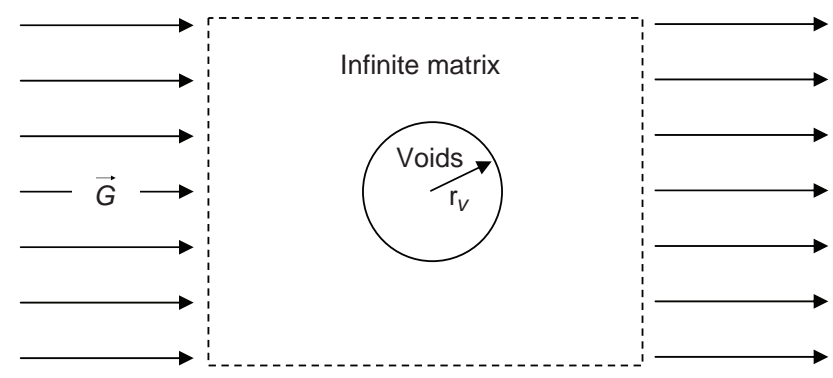

Figure 8. RVE of the porous matrix.

Finally, considering the interface conditions and the Equation (21), the following system of equations is obtained:

$$
\left[\begin{array}{ccc}
D_{m} r_{v}^{2} & -D_{m} & -D_{v} r_{v}^{2} \\
D_{e f f}^{m}-D_{m} & 0 & v_{v}\left(D_{m}-D_{v}\right) \\
\alpha_{m v} r_{v}^{2} & \alpha_{m v} & -r_{v}^{2}
\end{array}\right]\left[\begin{array}{c}
G \\
B_{m} \\
A_{v}
\end{array}\right]=\left[\begin{array}{l}
0 \\
0 \\
0
\end{array}\right]
$$

By making the determinant of (22) equal to zero, the expression of the matrix effective diffusivity is obtained:

$$
\frac{D_{e f f}^{m}}{D_{m}}=1+2 \alpha_{m v} v_{v} \frac{\left(D_{v} / D_{m}\right)-1}{\left(D_{v} / D_{m}\right)+1} .
$$

The moisture content gap parameter can be defined by the ratio:

$$
\alpha_{m v}=\frac{\rho_{w}}{M_{m} \rho_{m}\left(1-v_{v}\right)},
$$

where $\rho_{w}$ and $\rho_{m}$ are respectively the densities of water and matrix whereas $M_{m}$ is the moisture content at saturation in the matrix.

The moisture content at saturation $M_{m}$, for a 5208 epoxy resin subjected to a relative humidity of $100 \%$, is $5.9 \%$ according to Loos and Springer [14]. $\alpha_{m v}$ value is deduced from Equation (24). For values of void volume fractions varying from $1 \%$ to $5 \%$, there is no significant evolution of $\alpha_{m v}$. As a consequence, an average value $\alpha_{m v}=15$ will be assumed for the gap parameter in the considered range of voids volume fractions.

Figure 9 shows the matrix effective diffusivity as a function of the normalized voids diffusivity $D_{v} / D_{m}$ for different void volume fractions. We assume that the void diffusivity is higher than that of the matrix. The curves have the same departure point corresponding to $D_{v}=D_{m}=D_{e f f}^{m}$. The effective diffusivity is a growing function of $D_{v} / D_{m}$ while this very ratio remains weaker than around 10 . Beyond this point, $D_{\text {eff }}^{m}$ becomes nearly constant.

In order to estimate the effective diffusivity of a composite containing impermeable fibers, the matrix effective diffusivity is introduced in expression (11):

$$
\frac{D_{e f f}}{D_{m}}=\left[1+2 \alpha_{m v} v_{v} \frac{\left(D_{v} / D_{m}\right)-1}{\left(D_{v} / D_{m}\right)+1}\right] \frac{1-v_{f}}{1+v_{f}} .
$$


Figure 10 shows the effective diffusivity as a function of the fiber volume fraction for a porous composite material exhibiting $0,2,5$, and $10 \%$ void volume fractions. The fibers do not contribute to the diffusion process. Thus, an increase of the fiber volume fraction involves a decrease of the effective diffusivity.

\section{Four-phase Approach: Fiber, Void, Matrix, Equivalent Homogeneous Medium}

In this approach the void is modeled as a thin layer located between the fiber and the matrix (Figure 11).

In this case, the cellular diffusion problem through each layer is expressed as follows:

$$
\left\{\begin{array}{ll}
\operatorname{div} \vec{q}(y)=0 & \text { in each phase } V_{\alpha} \\
\vec{q}=-D \operatorname{grad} C & \text { in each phase } V_{\alpha} \\
\|\vec{q} \cdot \vec{n}\|=0 & \text { at interfaces } \\
\|C\| \neq 0 & \text { at interfaces }
\end{array} .\right.
$$

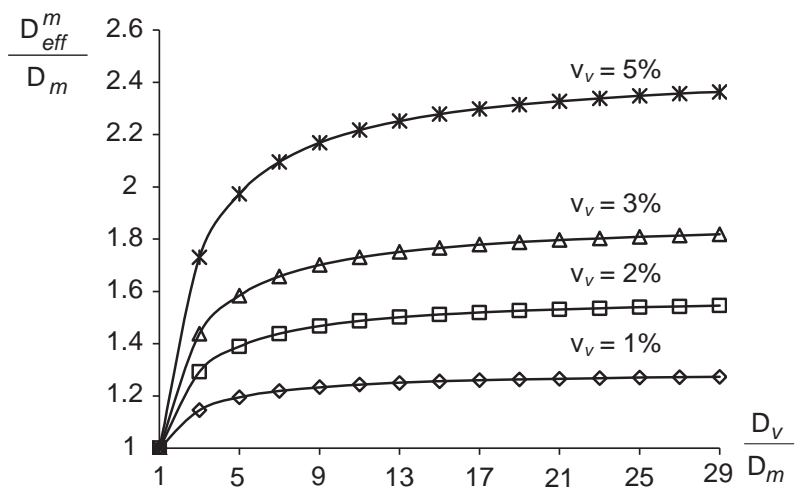

Figure 9. Dimensionless matrix effective diffusivity as a function of $D_{v} / D_{m}$.

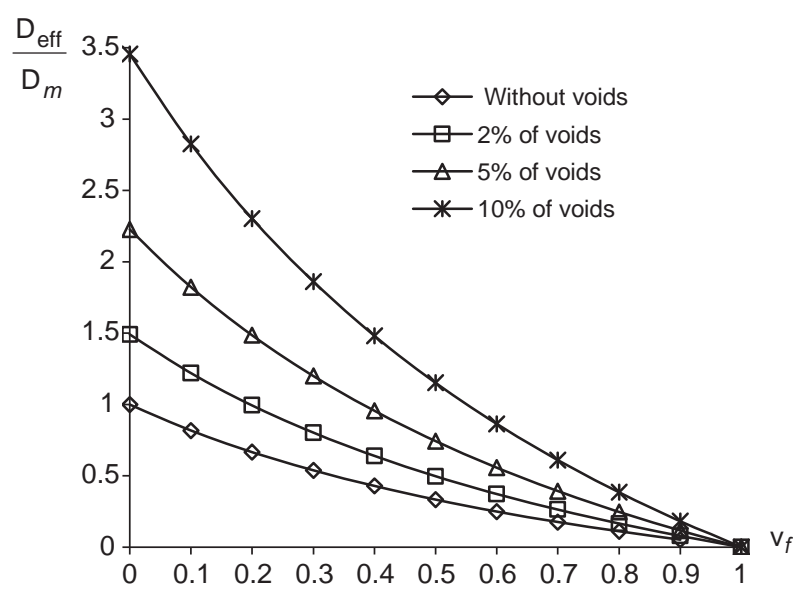

Figure 10. Void effects on the composite diffusivity in the case of impermeable fibers $\left(D_{v} / D_{m}=10, \alpha_{m v}=15\right)$. 
The solution fields respect the forms:

$$
\begin{cases}C(r, \theta)=A_{f} r \cos (\theta) & \text { in the fiber } \\ C(r, \theta)=\left(A_{v} r+\frac{B_{v}}{r}\right) \cos (\theta) & \text { in the voids } \\ C(r, \theta)=\left(A_{m} r+\frac{B_{m}}{r}\right) \cos (\theta) & \text { in the matrix } \\ C(r, \theta)=G r \cos (\theta) & \text { in the equivalent medium }\end{cases}
$$

where $A_{f}, A_{v}, A_{m}, B_{v}, B_{m}$, and $G$ are unknown constants.

The conditions of flux continuities and moisture content discontinuities assumed to hold at each interfaces involve the following set of equations:

$$
\left[\begin{array}{cccccc}
0 & 0 & \alpha_{f v} r_{f}^{2} & \alpha_{f v} & -r_{f}^{2} & 0 \\
0 & 0 & D_{v} r_{f}^{2} & -D_{v} & -D_{f} r_{f}^{2} & 0 \\
\alpha_{m v} r_{v}^{2} & \alpha_{m v} & -r_{v}^{2} & -1 & 0 & 0 \\
D_{m} r_{v}^{2} & -D_{m} & -D_{v} r_{v}^{2} & D_{v} & 0 & 0 \\
r_{m}^{2} & 1 & 0 & 0 & 0 & -r_{m}^{2} \\
D_{m} r_{m}^{2} & -D_{m} & 0 & 0 & 0 & -D_{e f f} r_{m}^{2}
\end{array}\right]\left[\begin{array}{c}
A_{m} \\
B_{m} \\
A_{v} \\
B_{v} \\
A_{f} \\
G
\end{array}\right]=\left[\begin{array}{c}
0 \\
0 \\
0 \\
0 \\
0 \\
0
\end{array}\right],
$$

where $\alpha_{f v}$ and $\alpha_{m v}$ are moisture content gaps corresponding to the voids/fiber and voids/ matrix interfaces, respectively. The knowledge of moisture content at saturation and diffusivities of composite and matrix leads to the determination of gap parameters and enables estimating the fiber diffusivity. By making the determinant of (28) equal to zero, one actually obtains the following expression of the normalized effective diffusivity:

$\frac{D_{e f f}}{D_{m}}=\frac{\left(1-k v_{f}\right)\left[1+k+\alpha_{f v}\left(D_{f} / D_{v}\right)(k-1)\right]+\alpha_{m v}\left(1+k v_{f}\right)\left[\left(D_{v} / D_{m}\right)(k-1)+\alpha_{f v}\left(D_{f} / D_{m}\right)(1+k)\right]}{\left(1+k v_{f}\right)\left[1+k+\alpha_{f v}\left(D_{f} / D_{v}\right)(k-1)\right]+\alpha_{m v}\left(k v_{f}-1\right)\left[\left(D_{v} / D_{m}\right)(1-k)-\alpha_{f v}\left(D_{f} / D_{m}\right)(1+k)\right]}$,

where, $k=\left(v_{v}+v_{f} / v_{f}\right), v_{v}$ and $v_{f}$ being the void and fiber volume fractions, respectively.

By introducing $\phi=\alpha_{m v}\left(D_{v} / D_{m}\right)$ and $\psi=\alpha_{f v}\left(D_{f} / D_{m}\right)$, Equation (29) becomes:

$$
\frac{D_{e f f}}{D_{m}}=\frac{\left(1-k v_{f}\right)\left[1+k+\alpha_{m v}(\psi / \phi)(k-1)\right]+\left(1+k v_{f}\right)\left[\phi(k-1)+\alpha_{m v} \psi(1+k)\right]}{\left(1+k v_{f}\right)\left[1+k+\alpha_{m v}(\psi / \phi)(k-1)\right]+\left(1-k v_{f}\right)\left[\phi(k-1)+\alpha_{m v} \psi(1+k)\right]}
$$

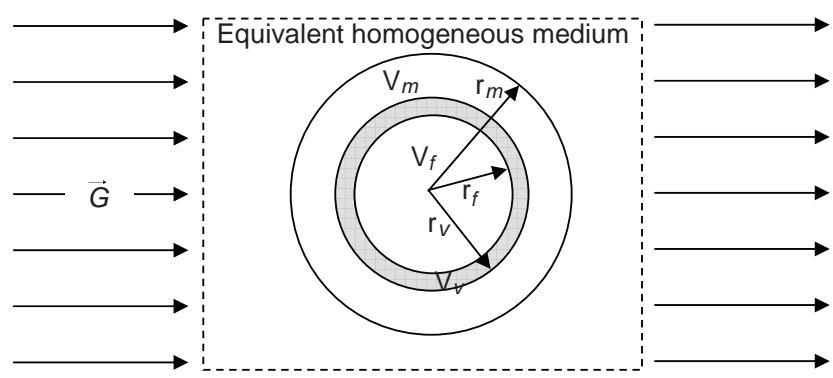

Figure 11. RVE of the four-phase approach. 
Assuming that moisture diffusion in void is faster than in any other constituents, the set of inequations $\alpha_{f v}\left\langle 1\right.$ and $\left.\alpha_{m v}\right\rangle 1$ is considered. According to the literature, $\alpha_{f v}=0.2$ (i.e., $=4.18$ ) would for instance correspond to an interface jute fiber/voids [15].

Figure 12 shows the evolution of the effective diffusivity of a composite material with permeable fibers in the case that $\psi=4.18$ and $\phi=150\left(D_{v} / D_{m}=10\right.$ and $\left.\alpha_{m v}=15\right)$ for various volume fractions of voids. A large increase of the diffusivity as a function of the rate of voids is obtained. For $2 \%$ of voids and a fiber volume fraction equal to $70 \%$, an increase of about $35 \%$ is observed. For $10 \%$ of voids and the same fiber volume fraction, the increase in terms of diffusivity is about $100 \%$. These values reveal the strong sensitivity of the effective diffusivity to the void volume fraction.

\section{IMPERMEABLE FIBERS}

The expression of the effective diffusion coefficient for composites with impermeable fibers is obtained by considering $D_{f}=0$ (i.e., $\psi=0$ ) in expression (30), which simplifies as follows:

$$
\frac{D_{e f f}}{D_{m}}=\frac{\left(1-k v_{f}\right)(1+k)+\phi\left(1+k v_{f}\right)(k-1)}{\left(1+k v_{f}\right)(1+k)+\phi\left(1-k v_{f}\right)(k-1)} .
$$

By putting $v_{v}=0$ (i.e. $k=1$ ) in (31), we recognize the expression (11) of the normalized effective diffusivity.

Figure 13 shows the effective diffusivity as a function of the fiber volume fraction for a composite material containing respectively $0,2,5$, and $10 \%$ in volume of voids. We note that the difference between the diffusion coefficients is proportional to the voids volume fraction. The difference observed between the effective diffusivities for nonporous and porous materials is more important than in the case of permeable fibers: for the volume fractions of fibers and voids respectively equal to 10 and $70 \%$, the effective diffusion coefficient is 26 times more important than that of void-free composite.

A decrease in the effective diffusion coefficient as a function of $v_{f}$, in the case of a nonporous material, is observed. The presence of voids in the case that impermeable fibers

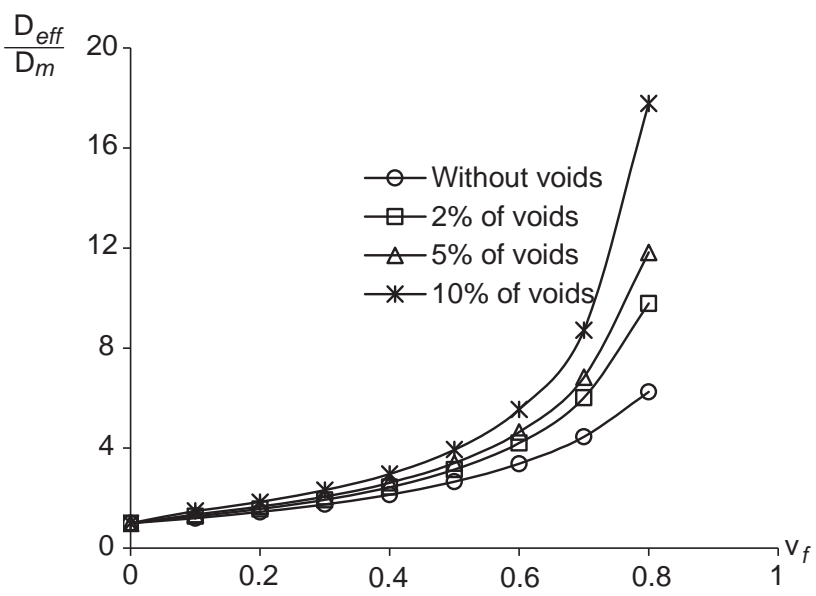

Figure 12. Void effects on the composite diffusivity in the case of permeable fibers ( $\phi=150, \xi=4.18$ ). 
are considered not only tend to reduce the damping effect of the fibers on the effective diffusivity but also undertakes the influence of the matrix on the diffusive behavior of the composite. Such a behavior does not occur anymore when the effective diffusivity of the composite material is calculated by using the effective diffusivity of porous matrix deduced from the diluted approach (Figure 10).

\section{Void Modeling with Self-consistent Model}

The basic idea of void modeling by the self-consistent model is to consider the composite RVE as consisting of three heterogeneous phases: fiber, void (represented by a thin layer surrounding the fiber), and the equivalent media (Figure 14).

In this case, the solution fields satisfy:

$$
\begin{cases}C(r, \theta)=A_{f} r \cos (\theta) & \text { in the fiber } \\ C(r, \theta)=\left(A_{v} r+\frac{B_{v}}{r}\right) \cos (\theta) & \text { in the void } \\ C(r, \theta)=\left(G r+\frac{B_{a c}}{r}\right) \cos (\theta) & \text { in the equivalent medium }\end{cases}
$$

where $A_{f}, A_{v}, B_{v}, B_{a c}$, and $G$ are unknown constants.

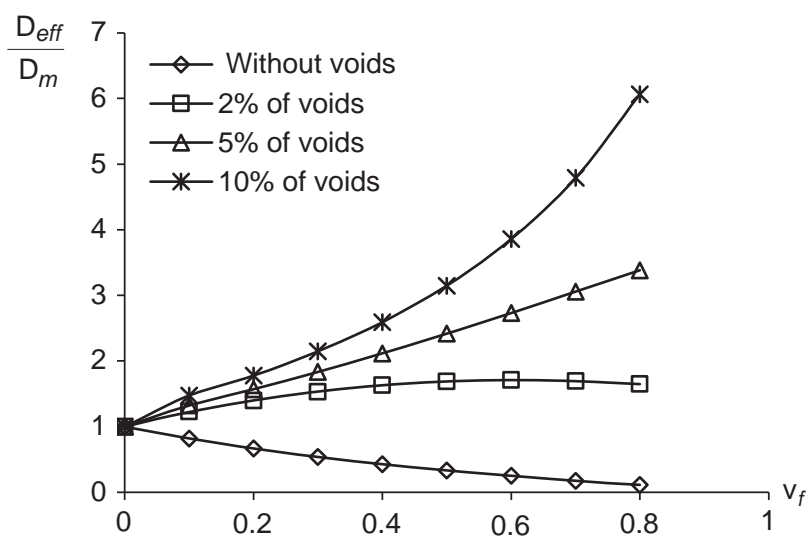

Figure 13. Void effects on the composite diffusivity in the case of impermeable fibers $(\phi=150)$.

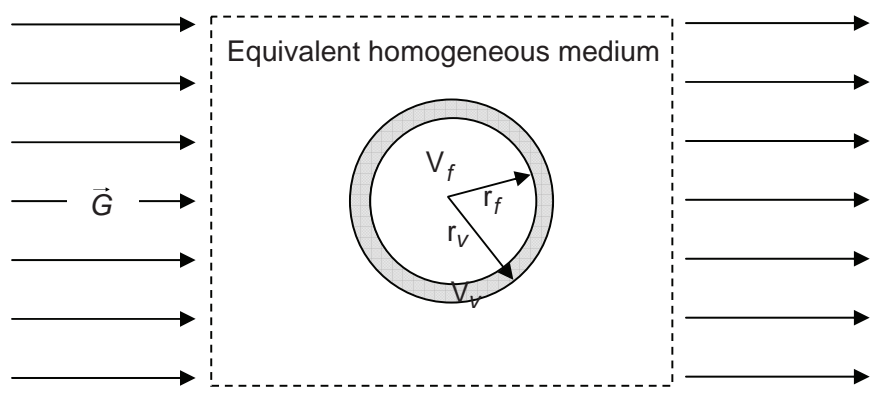

Figure 14. RVE of the the self-consistent model. 
Although the void and the equivalent medium are of different nature, moisture continuity is imposed between them since they are not at the same scale. In this case, the homogeneous equivalent behavior is defined as follows:

$$
D_{e f f}\langle\overrightarrow{\operatorname{grad}} C(y)\rangle=v_{f} D_{f}\langle\overrightarrow{\operatorname{grad}} C(y)\rangle_{V_{f}}+v_{m} D_{e f f}\langle\overrightarrow{\operatorname{grad}} C(y)\rangle_{V_{m}}+v_{v} D_{v}\langle\overrightarrow{\operatorname{grad}} C(y)\rangle_{V_{v}} .
$$

The conditions of flux continuities and moisture content continuity and discontinuities at the interfaces with the homogeneous equivalent behavior, lead to the following set of equations:

$$
\left[\begin{array}{ccccc}
r_{f}^{2} & -\alpha_{f v} r_{f}^{2} & -\alpha_{f v} & 0 & 0 \\
D_{f} r_{f}^{2} & -D_{v} r_{f}^{2} & D_{v} & 0 & 0 \\
0 & r_{v}^{2} & 1 & -r_{v}^{2} & -1 \\
0 & D_{v} r_{v}^{2} & -D_{v} & -D_{e f f} r_{v}^{2} & D_{e f f} \\
v_{f}\left(D_{m}-D_{f}\right) & v\left(D_{m}-D_{v}\right) & 0 & \left(D_{e f f}-D_{m}\right) & 0
\end{array}\right]\left[\begin{array}{c}
A_{f} \\
A_{v} \\
B_{v} \\
G \\
B_{a c}
\end{array}\right]=\left[\begin{array}{c}
0 \\
0 \\
0 \\
0 \\
0
\end{array}\right] .
$$

Making the system determinant equal to zero provides the expression of the effective diffusivity. The expression of composite diffusivity for impermeable fibers is obtained by considering $D_{f}=0$ and $\alpha_{f v}=0$ in the system determinant, then:

$$
\begin{aligned}
\frac{D_{e f f}}{D_{m}}= & \frac{1}{2(1+k)}\left[\frac{D_{v}}{D_{m}}\left(1+k\left(2 v_{v}-1\right)\right)+\left(1+k\left(1-2 v_{v}\right)\right)\right. \\
& \left.+\sqrt{4 \frac{D_{v}}{D_{m}}\left(k^{2}-1\right)+\left[\frac{D_{v}}{D_{m}}\left(1+k\left(2 v_{v}-1\right)\right)+1+k\left(1-2 v_{v}\right)\right]^{2}}\right]
\end{aligned}
$$

where $k=v_{v}+v_{f} / v_{f}$.

The fact that the expression of the diffusion coefficient of the composite is only a function of voids reveals a strong sensitivity to voids and implies that the composite diffusion behavior is completely governed by the voids.

Figure 15 shows the effective diffusivity deduced from the self-consistent model approach for a composite material with 1,5 , and $10 \%$ void fractions for a $k$-value corresponding to $v_{f}=0.7$. The diffusivity for the void-free composite is calculated from expression (17) according to an iterative process. The difference between the effective diffusivities for nonporous and porous materials becomes stronger as the void diffusivity increases. For $1 \%$ of voids and $D_{v} / D_{m}=10$, the effective diffusion coefficient is 4.4 times more important than that of the void-free composite. This reveals a high sensitivity to void volume fraction.

Figure 16 illustrates the effects due to the void volume fraction on the predicted effective diffusivity calculated through the various approaches previously described in the present work. The approach based on the porous matrix is the less sensitive to the voids volume fraction, whereas the strongest effects due to the presence of voids is obtained with the four-phase approach. The porous matrix and the four-phase approaches have the same departing point corresponding to the diffusivity value of a free-void composite at $v_{f}=0.7$ 


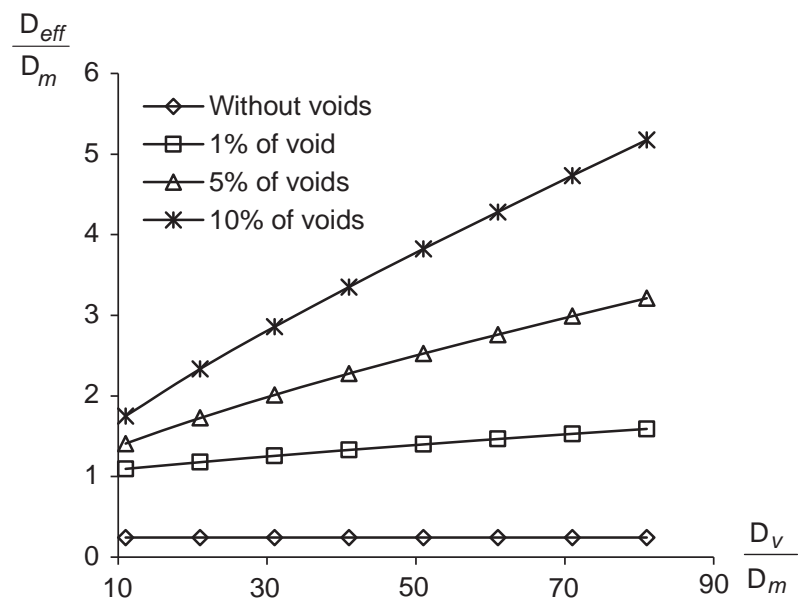

Figure 15. Void effects on the composite diffusivity in the case of impermeable fibers.

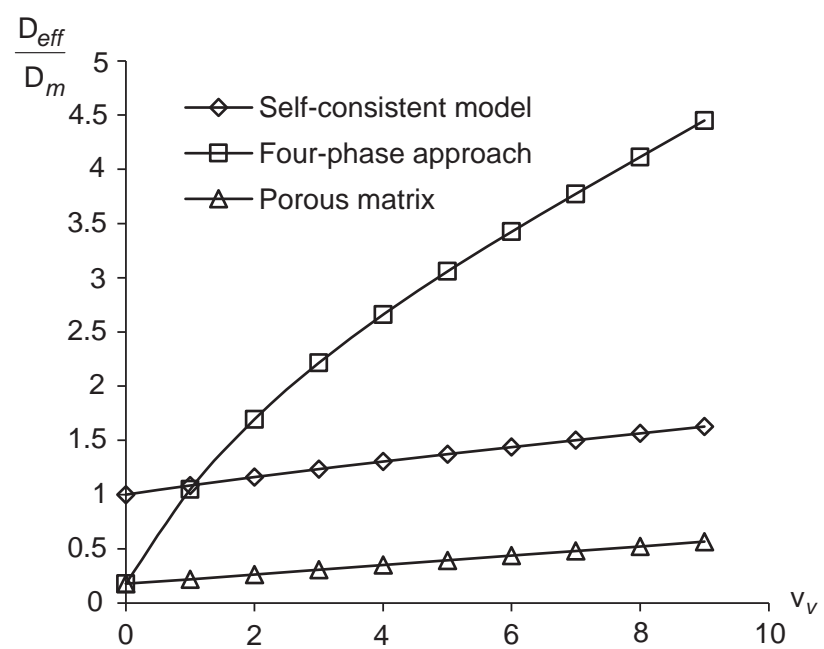

Figure 16. Void content effects on the composite diffusivity in the case of impermeable fibers $\left(D_{v} / D_{m}=10\right)$.

but the difference between both these approaches continuously increases with the void volume fraction.

\section{CONCLUSION}

New closed-form solutions are proposed for calculating the effective diffusivities of fiber-reinforced composite plies as functions of the proper diffusivities of the constituents, their volume fraction, and in the case that permeable fibers are considered, as a function of a representative parameter of the moisture content gap at the boundary between the constituents. These solutions also allow estimating the moisture diffusivity of a constituent, from the knowledge of the effective moisture diffusivity, through inverse approaches. A comparison with effective diffusivities calculated by using finite elements is 
also made and gives satisfactory results. A special attention is paid to the determination of the effective moisture diffusivity for composite materials containing voids, which are often met in practice in such materials. The influence of void content on the diffusion process is emphasized through several numerical applications showing the strong interaction between porosity and the effective moisture transport behavior of fiber-reinforced polymer matrix composite plies.

In further works, the coupling between the moisture diffusivities and the mechanical stress states will be numerically investigated. The correlation between the predicted and experimental results will be checked.

\section{REFERENCES}

1. Tsai, S.W. and Hahn, H.T. (1980). Introduction to Composite Materials, Westport, Conn.: Technomic Pub.

2. Springer, G.S. and Shen, C.H. (1976). Moisture Absorption and Desorption of Composite Materials, Journal of Composite Materials, 10: 2-20.

3. Jacquemin, F., Vautrin, A. (2002). Modelling of the Moisture Concentration Field due to Cyclical Hygrothermal Conditions in Thick Laminated Pipes, European Journal of Mechanics, 2: $845-855$.

4. Stastna, J. and De Kee, D. (1995). Transport Properties in Polymers, Lancaster, Pa.: Technomic Pub.

5. Hashin, Z. (1972). Theory of Fibre Reinforced Materials, NASA CR-1974.

6. Viéville, P. and Lipinski, P. (1994). Application du shéma autocohérent par étapes à la modélisation des propriétés viscoélastique des composites, In: $9^{\text {ème }}$ Journées Nationales Composites, Saint-Etienne, France.

7. Viéville, P. (1992). Influence des paramètres architecturaux sur les caractéristiques du bois à ses différentes échelles d'hétérogénéités, PhD Thesis of the Institut Polytechnique de Lorraine.

8. Whitcomb, J. and Tang, X. (2002). Micromechanics of Moisture Diffusion in Composites with Impermeable Fibres, Journal of Composite Materials, 36: 1093-1101.

9. Bond, D.A. (2005). Moisture Diffusion in a Fiber-reinforced Composite: Part I - Non-fickian Transport and the Effect of Fiber Spatial Distribution, Journal of Composite Materials, 39: 2113-2141.

10. Huang, H. and Talreja, R. (2005). Effects of Voids Geometry on Elastic Properties of Unidirectional Fibre Reinforced Composites, Composite Science and Technology, 65: 1964-1981.

11. Youssef, Z., Jacquemin, F., Gloaguen, D. and Guillén, R. (2007). Hygro-elastic Internal Stresses in Porous Composite Materials: A Multi-scale Analysis, Journal of Reinforced Plastics and Composites, 26: 1865-1880.

12. Harper, B.D., Staab, G.H. and Chen, R.S. (1987). A Note on the Effects of Voids Upon the Hygral and Mechanical Properties of AS4/3502 Graphite/Epoxy, Journal of Composite Materials, 21: 280-289.

13. Woo, M. and Piggot, M.R. (1988). Water Absorption of Resins and Composites: IV Water Transport in Fiber Reinforced Plastics, Journal of Composites Technology \& Research, 10: 20-24.

14. Loos, A.C. and Springer, G.S. (1981). Environmental Effects on Composite Materials, Lancaster, Pa.: Technomic Pub.

15. Rao, R.M., Chanda, M. and Balsubramanian, A. (1984). Factors Affecting Moisture Absorption in Polymer Composites Part 11: Influence of External Factors, Journal of Reinforced Plastic and Composites, 3: 246-251. 